\title{
MODIFICAÇÃO QUÍMICA DO AMIDO EXTRAÍDO DO RESÍDUO DO PROCESSAMENTO AGROINDUSTRIAL DA MANGA (Mangifera indica L.) VAR. UBÁ
}

\author{
C. A. FERRAZ ${ }^{1}$, R. L. SILVA ${ }^{2}$, G. C. FONTES $^{1}$, M. H. M. ROCHA-LEÃO ${ }^{1}$ \\ ${ }^{1}$ Universidade Federal do Rio de Janeiro, Escola de Química \\ ${ }^{2}$ Universidade Federal do Rio de Janeiro, Instituto de Química \\ E-mail para contato: gizelecf@yahoo.com.br
}

\begin{abstract}
RESUMO: O Brasil é um dos maiores produtores mundiais de manga e a maior parte da produção destina-se para a indústria de sucos, onde a variedade Ubá se destaca. Neste processamento é gerado um volume elevado de resíduos, composto por cascas e sementes, que podem ser aproveitados agregando valores ao agronegócio. Dessa forma, o objetivo do presente trabalho foi modificar quimicamente o amido extraído da amêndoa da semente da manga através da reação de esterificação com o anidro octenil-succínico (OSA). Inicialmente, foi determinada a composição centesimal da farinha da amêndoa da manga, e em seguida, a extração do amido com bissulfito de sódio $(0,16 \%)$ obtendo-se um rendimento de $45 \%$ em massa, com teor de amido de 99,27\%. O amido extraído foi submetido à reação de modificação com rendimento de $77,74 \%$. O grau de substituição foi de 0,04 , valor esse similar a amidos comerciais modificados. Portanto, o amido extraído do resíduo da manga apresenta grande potencial de aplicação como biopolímero.
\end{abstract}

\section{INTRODUÇÃO}

Atualmente, grande parte dos resíduos agroindustriais do processamento de frutas para a obtenção de sucos é descartada no solo sem qualquer tratamento prévio. Estas toneladas de subprodutos desperdiçados podem ter valor agregado, através de investigação científica e tecnológica (VIEIRA, 2007). O aproveitamento destes resíduos como ingredientes para incorporação em alimentos alternativos ou na aplicação em novas tecnologias consiste em uma alternativa bem sucedida para a indústria de alimentos.

A manga é um fruto largamente consumido nos países de clima tropical, sendo o Brasil um dos maiores produtores mundiais destinando grande parte de sua produção para a indústria de sucos, onde a cultivar Ubá se destaca. Entretanto, neste processamento cerca de 40 e $60 \%$ da matéria-prima constituem-se de resíduos, sendo de aproximadamente 12 a $15 \%$ de cascas e de 15 a $20 \%$ de sementes (RAMOS et al, 2005).

O amido é certamente um dos materiais mais versáteis para uso potencial em polímeros. Ele pode ser usado na produção de polímeros sintéticos, na produção de biopolímeros através de processos fermentativos ou ainda ser hidrolisado e empregado como monômero ou oligômero (BRITO, 2011). Em relação aos biopolímeros, os plásticos de amido são atualmente os mais importantes no mercado, onde a maioria é usada no setor de embalagens, incluindo filmes solúveis para embalagens industriais e filmes para sacos. As 
aplicações emergentes são no setor automobilístico, em eletrônicos e eletrodomésticos e em outras aplicações duráveis (SHEN et al., 2009). Dessa forma, o objetivo do presente trabalho foi modificar quimicamente o amido extraído da amêndoa da semente da manga através da reação de esterificação com o anidro octenil-succínico (OSA).

\section{MATERIAIS E MÉTODOS}

\subsection{Extração do amido da farinha da amêndoa da semente da manga}

As mangas (Mangifera indica L.) Var. Ubá foram adquiridas em mercado local na cidade de Viçosa (MG), sendo posteriormente descascadas e despolpadas para a retirada da semente. Para prensagem da casca da semente foi utilizado um torno mecânico de bancada $\left(\right.$ Motomil $^{\circledR}$ ), resultando na obtenção da amêndoa sendo esta posteriormente cominuída em um moinho de facas tipo Willye (Tecnal TE -680).

O fluxograma do processo de extração do amido foi ilustrado na Figura 1. A metodologia adotado foi feita segundo o procedimento descrito por Guraya et al. (2004) com modificações.

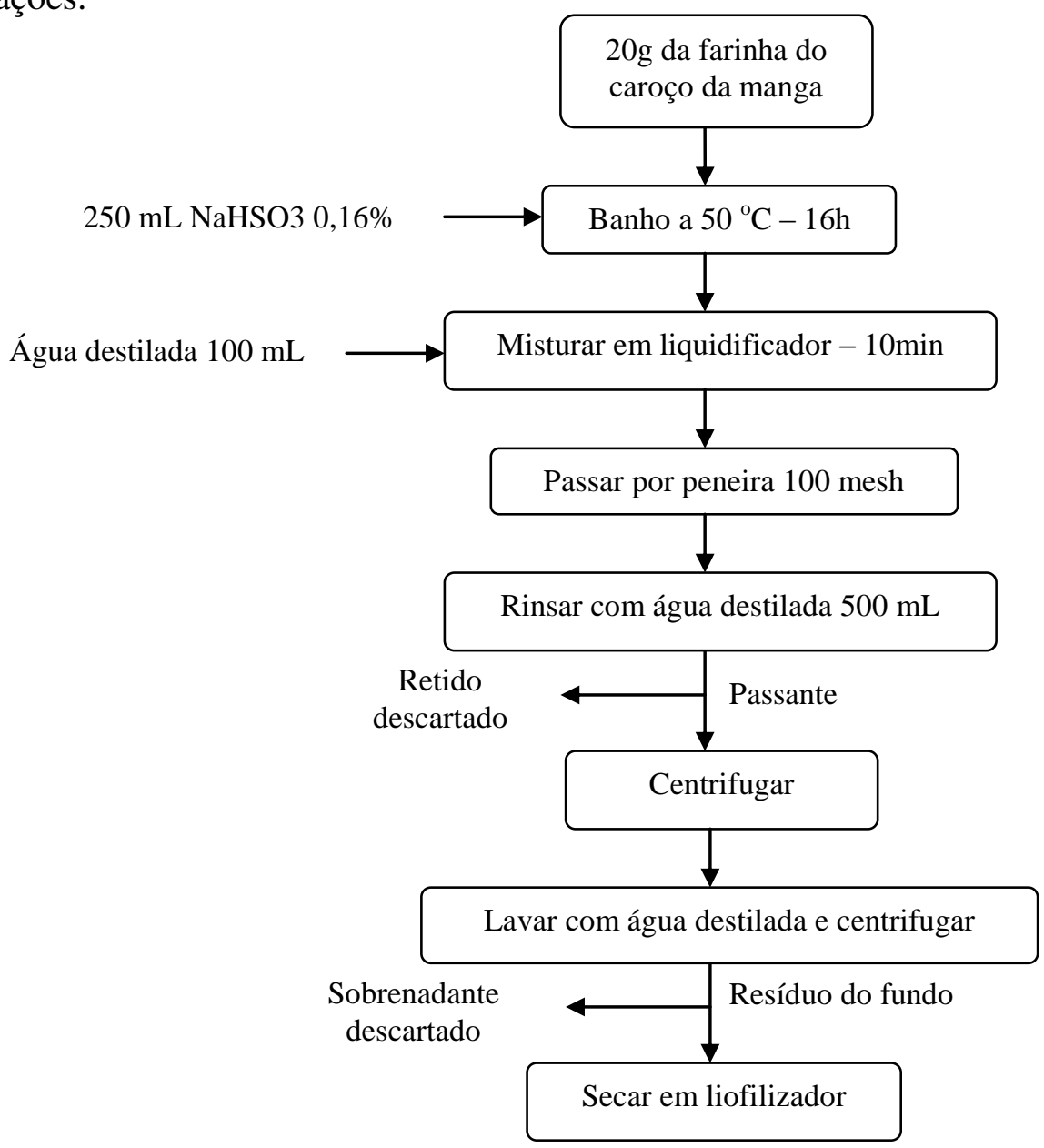

Figura 1 - Fluxograma da metodologia de extração. 
O rendimento da extração do amido foi calculado segundo a Equação 1.

$$
\text { Rendimento }=\frac{\text { Massa final }}{\text { Massa inicial }} \times 100
$$

Uma das modificações realizadas foi o desengraxe de parte da matéria prima usando um extrator de Soxhlet e hexano como solvente por 16 horas a $75^{\circ} \mathrm{C}$. A segunda modificação esta em centrifugar todo o passante após repouso de 1 hora sem retirar o sobrenadante.

\subsection{Composição centesimal da amêndoa da manga}

A análise físico-química da composição centesimal da farinha da amêndoa da manga bruta foi feita pelo Laboratório de Bromatologia da Faculdade de Farmácia da UFRJ usando os métodos descritos em Normas Analíticas do Instituto Adolfo Lutz (2005) e Official Methods of Analysis of AOAC International (1995). Os seguintes parâmetros foram determinados: teor de amido, de lipídios, de cinzas, de proteínas, de carboidratos totais (Nifext) e de umidade.

\subsection{Quantificação do amido na farinha da amêndoa da manga e das amostras de amido extraído}

A quantificação do amido da farinha da amêndoa bruta e do amido extraído foi feita pelo Laboratório de Bromatologia da Faculdade de Farmácia da UFRJ segundo o método descrito pela AOAC (Official Methods of Analysis of AOAC International, 1995).

\subsection{Modificação química do amido extraído}

A succinilação do amido foi feita em meio básico segundo o procedimento descrito por Song et al. (2006). $7 \mathrm{~g}$ do amido extraído da amêndoa da manga foram suspensos em água destilada numa proporção de $35 \%$ (p/p) com agitação e $\mathrm{pH}$ 8,5. Uma quantidade de $3 \%$ (p/p com base no amido) do Amido de milho OSA comercial - Capsul ${ }^{\circledR}$ (OSA Corn Products International, Inc., Illinois, Estados Unidos da América), foi diluída 3 vezes em álcool etílico absoluto e adicionada lentamente. A reação durou no total $4 \mathrm{~h}$ e foi conduzida a $35^{\circ} \mathrm{C}$ em um banho-maria com agitação utilizando $\mathrm{HCl} \mathrm{3 \%}(\mathrm{p} / \mathrm{p})$ para parar a reação. A mistura foi centrifugada a $1575 \mathrm{~g}$ por 10 minutos e lavada por 2 vezes com água destilada e álcool etílico $70 \%$. O resíduo foi seco em uma estufa a $35^{\circ} \mathrm{C}$.

\subsection{Determinação do grau de substituição (GS)}

O grau de substituição foi realizado por uma titulação alcalina, segundo o procedimento de Kweon et al. (2001). Primeiramente, $1 \mathrm{~g}$ de amido foi disperso em $25 \mathrm{~mL}$ de uma solução $2,5 \mathrm{M} \mathrm{HCl} /$ álcool isopropílico e agitado por 30 minutos. A dispersão foi filtrada em um funil de separação a vácuo e o resíduo lavado com $100 \mathrm{~mL}$ de álcool isopropílico. O retentado foi em seguida disperso em $300 \mathrm{~mL}$ de água deionizada e aquecido em um banho-maria com água fervendo por 20 minutos para a gelatinização do grânulo de amido. A dispersão foi então titulada com uma solução de $0,1 \mathrm{M}$ de $\mathrm{NaOH}$. O GS foi determinado de acordo com a Equação 2. 


$$
G S=\frac{0,162 \times \frac{A \times N}{P}}{1-\left[0,101 \times \frac{A \times N}{P}\right]}
$$

em que: $\mathrm{A}=$ volume de $\mathrm{NaOH}$ usado na titulação, $\mathrm{N}=$ normalidade da solução de $\mathrm{NaOH}$ e $\mathrm{P}$ $=$ peso do amido seco.

Durante a titulação, o amido é passível de sofrer alguma degradação durante o tratamento, assim há a necessidade de obter um valor branco pela titulação do amido não substituído (SWEEDMANA et al., 2013). A titulação foi realizada (em triplicada) nas seguintes amostras de amido de manga nativo e modificado e de amido de milho comercial (Maizena $^{\circledR}$ ) e amido OSA comercial (Capsul ${ }^{\circledR}$ ) para comparação.

\section{RESULTADOS E DISCUSSÕES}

\subsection{Composição centesimal da farinha da amêndoa da manga}

Inicialmente, foi realizada a determinação da composição centesimal e teor de amido da farinha da amêndoa da manga. O resultado está apresentado na Tabela 1.

Tabela 1 - Composição centesimal da farinha da amêndoa bruta.

\begin{tabular}{cc} 
Componente & $(\boldsymbol{\%})$ \\
\hline Amido & $78,42( \pm 0,00)$ \\
Umidade & $4,26( \pm 0,08)$ \\
Lipídios & $10,78( \pm 0,16)$ \\
Cinzas & $1,96( \pm 0,05)$ \\
Proteína & $4,57( \pm 0,61)$ \\
Carboidratos totais (Nifext) & 78,43 \\
\hline
\end{tabular}

Mendes et. al (2012), encontraram em amostras de farinha da amêndoa da manga variedade Tommy Atkins, cerca de $71 \%$ de amido. Os resultados obtidos mostram que a amêndoa da manga variedade Ubá apresenta um alto teor de amido quando comparado com outras variedades de manga. Provavelmente, o acúmulo de amido nas sementes está relacionado as diferenças de genótipo de cada variedade, fatores climático e de solo as quais cada uma são submetidas.

Com relação à composição de proteína e lipídios, o resultado obtido foi similar ao obtido por Vieira et al. (2009) que analisou a mesma variedade da farinha da amêndoa da manga, encontrando $3,87 \%$ e $12,18 \%$, respectivamente. A quantidade de cinzas obtida foi igual à composição obtida por esses autores, $1,96 \%$ de cálcio, fósforo e compostos minerais. 


\subsection{Extração do amido}

Para o início do procedimento de extração do amido, as amostras de farinha de amêndoa, com desengraxe e sem desengraxe, foram suspensas em solução de $\mathrm{NaHSO}_{3}(0,16$ $\%$ ), em triplicata. O rendimento em massa de cada extração pode ser encontrado na Tabela 2. Não houve diferenças significativas em relação ao rendimento final dos quatro tipos de amostras, confirmados pelo teste estatístico de Fischer. Assim, as etapas de desengraxe e repouso podem ser subtraídas da metodologia de extração.

Tabela 2 - Rendimento da extração de amido para os quatro tipos de amostra de farinha de amêndoa de manga, variedade "Ubá, na ausência ou presença das etapas de desengraxe e repouso.

\begin{tabular}{ccc}
\hline Amostra & Massa final (g) & Rendimento (\%) \\
\hline Com desengraxe e com repouso & 8,04 & $40,20 \%$ \\
Com desengraxe e sem repouso & 8,59 & $42,95 \%$ \\
Sem desengraxe e com repouso & 7,72 & $38,60 \%$ \\
Sem desengraxe e sem repouso & 8,53 & $42,57 \%$ \\
\hline
\end{tabular}

O teor de amido encontrado nos quatro tipos de amostra pode ser encontrado na Tabela 3. Observa-se um elevado teor de amido, mostrando que as 3 lavagens com água realizadas durante a extração foram suficientes para purificar o amido.

Tabela 3 - Teor de amido para os quatro tipos de amostra de farinha de amêndoa de manga, variedade "Ubá, na ausência ou presença das etapas de desengraxe e repouso após a extração

\begin{tabular}{ccc}
\hline Amostra & Teor de amido $(\%)$ & Coeficiente de variação (\%) \\
\hline Sem desengraxe e com repouso & $96,11( \pm 1,97)$ & 2,05 \\
Sem desengraxe e sem repouso & $99,27( \pm 0,10)$ & 0,10 \\
Com desengraxe e com repouso & $96,93( \pm 0,10)$ & 0,10 \\
Com desengraxe e sem repouso & $97,85( \pm 2,00)$ & 2,04 \\
\hline
\end{tabular}

Os resultados encontrados mostraram que o amido extraído das amostras desengraxadas não apresentou um grau de pureza maior que as provenientes das amostras não desengraxadas, assim as etapas de desengraxe e repouso foram eliminadas da metodologia de extração. Com essas modificações a metodologia ficou mais limpa, devido a não utilização de solventes orgânicos, e de maneira contínua, ou seja, com maior facilidade de ser implementada industrialmente. A amostra sem desengraxe e sem repouso foi escolhida para dar continuidade ao trabalho sendo submetida a modificação química.

\subsection{Modificação química do amido}


O amido extraído da manga foi modificado pela esterificação das hidroxilas do amido com o ácido octenilsuccinato anidro resultando, com isso, em um amido hidrofobicamente modificado. As reações de succiniliação foram realizadas em triplicata. O rendimento médio das reações foi de $77,74 \%( \pm 0,04)$.

A eficiência da reação é geralmente calculada usando-se o grau de substituição, calculado através de uma titulação, obtendo valores médios de 0,4. Esse valor foi similar ao GS encontrado no amido modificado comercial, Capsul ${ }^{\circledR}$. O GS depende do conteúdo de amilose e das condições de reação, assim há uma grande variação de resultados encontrados na literatura. Song et al. (2006) encontraram o grau de substituição de 0,018 utilizando as mesmas condições de reação, porém usando a matéria prima amido de arroz. Han e Lim (2012) encontraram GS de 0,025 na reação feita em condições similares também utilizando o amido de arroz.

\section{CONCLUSÃO}

A composição centesimal da farinha da amêndoa da manga variedade Ubá obteve resultados similares aos encontrados na literatura. A extração do amido utilizando o bissulfito de sódio $(0,16 \%)$ resultou em um rendimento de $45 \%$ em massa, com teor de amido de 99,27 $\%$. O amido extraído foi submetido à reação de modificação com rendimento de 77,74 \%. O grau de substituição foi de 0,04 , valor esse similar a amidos comerciais modificados. Portanto, o amido extraído do resíduo da manga apresenta grande potencial de aplicação como biopolímero. Novos estudos são necessários a fim de otimizar a metodologia de extração do amido.

\section{REFERÊNCIAS BIBLIOGRÁFICAS}

BRITO, G. F; AGRAWAL, P.; ARAÚJO, E. M.; MÉLO, T. J. A. Biopolímeros, Polímeros Biodegradáveis e Polímeros Verdes. REMAP, v.6, n.2, p. 127-13, 2011.

GURAYA, H. S.; KAUR, M.; SANDHU, K. S; SINGH, N. Physicochemical, morphological, thermal, and rheological properties of starches separated from kernels of some Indian mango cultivars (Mangifera indica L.). Food Chem, v. 85, p. 131-140, 2004.

KWEON, D. K., CHOI, J. K., KIM, E. K., LIM, S. T. Adsorption of divalent metal ions by succinylated and oxidized corn starches. Carbohydr Polym, v. 46, p. 171-177, 2001.

MENDES, M.L.M.; BORA, P.S.; RIBEIRO, A.P.L. Propriedades morfológicas e funcionais e outras características da pasta do amido nativo e oxidado da amêndoa do caroço de manga. (Mangifera indica L.), variedade Tommy Atkins. Rev Inst Adolfo Lutz. v.71, n.1, p. 76-84, 2012

RAMOS, A. M.; COUTO, F. A. A.; RESENDE, P. M. Manga ubá: boas práticas agrícolas para produção destinada à agroindústria. VIÇOSA: ED. UFV, 2005. 64p.

SHEN, L., HAUFE, J., PATEL, M. K. Product overview and market projection of emerging bio-based plastics - PRO-BIP 2009 Final report.

SONG, X., HE, G., RUAN, H., CHEN, Q. Preparation and Properties of Octenyl Succinic Anhydride Modified Early Indica Rice Starch. Starch/Stärke. v. 58, p. 109-117, 2006.

SWEEDMANA, M. C.,TIZZOTTI, M. J., SCHAFERB, C., GILBERTA, R. G. Structure and physicochemical properties of octenyl succinic anhydride modified starches: a review. Carbohydr Polym, 92, 905-920, 2013. 
VIEIRA, P.A.F.; Caracterização dos resíduos da manga (Mangifera indica L.) e efeitos sobre o desempenho e os parâmetros bioquímicos em frangos de corte. Dissertação (Mestrado em Bioquímica Agrícola). 2007. 92p. Universidade Federal de Viçosa, Minas Gerais, 2007.

VIEIRA, P. A. F., QUEIROZ, J. H., VIEIRA, B. C., MENTES, F., Q., BARBOSA, A., A., MULLER, E., S., SANT’ANA, R. C. O., MORAES, G. H. K. Caracterização química do resíduo do processamento agroindustrial da manga (Mangifera indica L.) Var. ubá. Alim. Nutr., v. 20, n. 4, p. 617-623, 2009. 